\title{
From triply charmed dibaryons to pentaquark states: A model-independent way to determine the spins of the $P_{c}(4440)$ and $P_{c}(4457)$
}

\author{
Ya-Wen Pan, ${ }^{1}$ Ming-Zhu Liu $\odot,{ }^{1}$ Fang-Zheng Peng, ${ }^{1}$ Mario Sánchez Sánchez, ${ }^{2}$ \\ Li-Sheng Geng $\odot,{ }^{3,4, *}$ and Manuel Pavon Valderrama ${ }^{1, \dagger}$ \\ ${ }^{1}$ School of Physics, Beihang University, Beijing 100191, China \\ ${ }^{2}$ Centre d'Études Nucléaires, CNRS/IN2P3, Université de Bordeaux, 33175 Gradignan, France \\ ${ }^{3}$ School of Physics and Beijing Key Laboratory of Advanced Nuclear Materials and Physics and Beijing \\ Advanced Innovation Center for Big Data-based Precision Medicine, \\ Beihang University, Beijing 100191, China \\ ${ }^{4}$ School of Physics and Microelectronics, Zhengzhou University, Zhengzhou, Henan 450001, China
}

(Received 31 July 2019; revised 12 December 2019; accepted 8 July 2020; published 27 July 2020)

The LHCb Collaboration has recently observed three narrow pentaquark states-the $P_{c}(4312)$, $P_{c}(4440)$, and $P_{c}(4457)$ - that are located close to the $\bar{D} \Sigma_{c}$ and $\bar{D}^{*} \Sigma_{c}$ thresholds. Among the so-far proposed theoretical interpretations for these pentaquarks, the molecular hypothesis seems to be the preferred one. Nevertheless, in the molecular picture, the spins of the $P_{c}(4440)$ and $P_{c}(4457)$ have not been unambiguously determined yet. In this paper, we point out that heavy-antiquark-diquark symmetry induces a model-independent relation between the spin splitting in the masses of the $P_{c}(4440)$ and $P_{c}(4457) \bar{D}^{*} \Sigma_{c}$ pentaquarks and the corresponding splitting for the $0^{+}$and $1^{+} \Xi_{c c} \Sigma_{c}$ triply charmed dibaryons. This is particularly relevant owing to a recent lattice-QCD prediction of the $1^{+}$triply charmed dibaryon, which suggests that a calculation of the mass of its $0^{+}$partner might be within reach. This, in turn, would reveal the spins of the $P_{c}(4440)$ and $P_{c}(4457)$ pentaquarks, providing a highly nontrivial test of heavy-quark symmetry and the molecular nature of the pentaquarks. Furthermore, the molecular interpretation of the hidden-charm pentaquarks implies the existence of a total of ten triply charmed dibaryons as $\Xi_{c c}^{(*)} \Sigma_{c}^{(*)}$ molecules, which, if confirmed in the lattice, will largely expand our understanding of the nonperturbative strong interaction in the heavy-quark sector.

DOI: 10.1103/PhysRevD.102.011504

Recently, the LHCb Collaboration has reported the observation of three pentaquark states [1] - the $P_{c}(4312)$, $P_{c}(4440)$, and $P_{c}(4457)$ - very close to two meson-baryon thresholds, the $\bar{D} \Sigma_{c}$ in the case of the $P_{c}(4312)$ and the $\bar{D}^{*} \Sigma_{c}$ in the case of the $P_{c}(4440)$ and $P_{c}(4457)$. This interesting coincidence has been promptly interpreted by a series of theoretical works [2-8] as evidence for their molecular nature, a hypothesis which is further fueled by the predictions a few years ago of the existence of these objects [9-14]. In addition to the molecular picture, there are other explanations, e.g., hadrocharmonium [15] or compact pentaquark states [16-18].

\footnotetext{
*lisheng.geng@buaa.edu.cn

mpavon@buaa.edu.cn
}

Published by the American Physical Society under the terms of the Creative Commons Attribution 4.0 International license. Further distribution of this work must maintain attribution to the author(s) and the published article's title, journal citation, and DOI. Funded by SCOAP .
However, the molecular interpretation cannot unambiguously determine the spins of the $P_{c}(4440)$ and $P_{c}(4457)$, which can be either $1 / 2$ or $3 / 2$. Initial studies showed a preference for the $P_{c}(4440)$ and $P_{c}(4457)$ being identified with $J=1 / 2$ and $3 / 2$ molecules, respectively $[3,4,6]$, while some recent theoretical works prefer the opposite identification [19-21].

A conclusive answer may demand larger statistics from the experiments. Given this situation, one might turn to lattice QCD for a model-independent answer. But mesonbaryon systems are difficult to study directly in the lattice, more so if there are coupled channels. After the 2015 pentaquark peaks [22], there have been only two lattice-QCD studies on $J / \psi N$ and $\eta_{c} N$ interactions [23,24], neither of which found pentaquarks. These studies, which were performed within a single-channel approximation, concluded that further calculations including coupled channels were needed.

To circumvent these difficulties, we propose to learn more about the $\bar{D}^{(*)} \Sigma_{c}^{(*)}$ pentaquarks from the $\Xi_{c c}^{(*)} \Sigma_{c}^{(*)}$ dibaryons. Both objects are connected by heavy-antiquarkdiquark symmetry (HADS). 
Hadronic molecules containing heavy quarks $(c, b)$ are constrained by heavy-quark symmetry ${ }^{1}$ (HQS) $[25,26]$, which can be exploited to predict unobserved states [27-30] or explain relations among experimentally known states [31-33]. Two manifestations of HQS are relevant for the present work: heavy-quark-spin symmetry (HQSS) (the heavy-hadron interaction is independent of the orientation of the heavy-quark spin) and HADS [34] (a pair of heavy quarks behaves like a heavy antiquark). Regarding the pentaquark states, HQSS predicts the existence of seven molecular states $[4,6,11,19,20,35]$ - from HQSS and the $\mathrm{LHCb}$ pentaquarks [1], one expects four more unobserved hidden-charm pentaquarks. ${ }^{2}$

But the discovery of the $\mathrm{LHCb}$ pentaquarks leads to more consequences. From HADS, we expect the lightquark structures of the $\bar{D}^{(*)}$ and the $\Xi_{c c}^{(*)}$ to be almost identical: If the $\bar{D}^{(*)} \Sigma_{c}^{(*)}$ system binds, the $\Xi_{c c}^{(*)} \Sigma_{c}^{(*)}$ system should also bind. Specifically, we can update the triplycharmed-dibaryon spectrum, from the four dibaryons originally predicted in Ref. [37] to ten dibaryons in the present manuscript. As we will show, this could help to determine the spins of the $P_{c}(4440)$ and $P_{c}(4457)$. Besides, it seems much easier for lattice QCD to study the $\Xi_{c c}^{(*)} \Sigma_{c}^{(*)}$ systems than the $\bar{D}^{(*)} \Sigma_{c}^{(*)}$ systems, which up to now lattice-QCD studies have not been able to simulate. On the other hand, a recent lattice-QCD study [38] has reported the likely existence of a triply charmed $\Xi_{c c} \Sigma_{c}$ dibaryon with spin parity $J^{P}=1^{+}$, isospin $I=1 / 2$, and binding energy $B_{2}=(8 \pm 17) \mathrm{MeV}$. Here, we predict this dibaryon to have a binding energy of $B_{2} \sim(15-30) \mathrm{MeV}$, compatible with the results of Ref. [38]. More importantly—as we will show-if lattice QCD is able to calculate the mass of the $J^{P}=0^{+}$configuration or the mass splitting between the $0^{+}$and $1^{+} \Xi_{c c} \Sigma_{c}$ dibaryons, it will provide a modelindependent determination of the spins of the $P_{c}(4440)$ and $P_{c}(4457)$.

Now we explain how to predict the binding energies of the triply charmed dibaryons. For this, following Refs. [4,39], we construct a contact-range effective field theory (EFT) that respects HQS. This assumes that pentaquarks and dibaryons are nonrelativistic bound states whose size is considerably larger than the interaction range. The leadingorder Lagrangian describing the $\bar{D}\left(\bar{D}^{*}\right)$ and $\Sigma_{c}\left(\Sigma_{c}^{*}\right)$ interaction and satisfying HQS can be easily obtained by introducing the superfields $[40,41]$

$$
H_{c}=\frac{1}{\sqrt{2}}\left(D+\vec{\sigma} \cdot \vec{D}^{*}\right), \quad \vec{S}_{c}=\frac{1}{\sqrt{3}} \vec{\sigma} \Sigma_{c}+\vec{\Sigma}_{c}^{*},
$$

\footnotetext{
${ }^{1}$ Conversely for the light quarks $(u, d, s)$ we have SU(3)-flavor and chiral symmetries, which originate from the observation that light quarks are indeed light.

${ }^{2}$ A recent analysis of the $2019 \mathrm{LHCb}$ data [36] showed that the $\bar{D} \Sigma_{c}^{*}$ state has, in fact, been seen, though only with a statistical significance of $1.7 \sigma$.
}

so that

$\mathcal{L}_{P}=C_{a} \operatorname{Tr}\left[H_{c}^{\dagger} H_{c}\right] \vec{S}_{c} \cdot \vec{S}_{c}^{\dagger}+C_{b} \sum_{i=1}^{3} \operatorname{Tr}\left[H_{c}^{\dagger} \sigma_{i} H_{c}\right] \vec{S}_{c} \cdot\left(J_{i} \vec{S}_{c}^{\dagger}\right)$,

with $\sigma_{i}\left(J_{i}\right)$ the three spin-1/2(1) matrices. For the $\Xi_{c c}\left(\Xi_{c c}^{*}\right)$, the superfield reads

$$
\vec{T}_{c c}=\frac{1}{\sqrt{3}} \vec{\sigma} \Xi_{c c}+\vec{\Xi}_{c c}^{*}
$$

while the Lagrangian can be deduced from HADS and Eq. (2) by replacing the traces $[39,42]$

$$
\begin{aligned}
\mathcal{L}_{D}= & C_{a} \vec{T}_{c c}^{\dagger} \cdot \vec{T}_{c c} \vec{S}_{c} \cdot \vec{S}_{c}^{\dagger} \\
& +C_{b} \sum_{i=1}^{3} \vec{T}_{c c}^{\dagger} \cdot\left(\sigma_{i} \vec{T}_{c c}\right) \vec{S}_{c} \cdot\left(J_{i} \vec{S}_{c}^{\dagger}\right) .
\end{aligned}
$$

From Eqs. (2) and (4), we find the potentials of Table I. We consider only contact-range interactions, as previous studies of two-heavy-hadron systems $[43,44]$ indicate that pion exchanges are perturbative in the charm sector.

The potentials of Table I are subject to a certain degree of uncertainty. They are derived from HQS, which is exact only in the limit of infinite heavy-quark masses, $m_{Q} \rightarrow \infty$. For finite $m_{Q}$, we expect small violations, which for HQSS are $\mathcal{O}\left(\Lambda_{\mathrm{QCD}} / m_{Q}\right)$ with $\Lambda_{\mathrm{QCD}} \sim(200-300) \mathrm{MeV}$ the QCD scale; i.e., we expect a $15 \%$ uncertainty in the charm sector for the pentaquark potentials. For HADS, the uncertainty is $\mathcal{O}\left(\Lambda_{\mathrm{QCD}} /\left(m_{Q} v\right)\right)$ [34], with $v$ the expected velocity for the heavy-quark pair. From the estimation of Ref. [45], $m_{Q} v \sim$ $0.8 \mathrm{GeV}$ for a charm quark pair, we arrive at a (25-40)\% uncertainty for HADS, consistent with the recent latticeQCD calculations [46-49] of the mass splitting between the

TABLE I. The lowest-order contact range potentials for the heavy-antimeson-heavy-baryon and doubly-heavy-baryonheavy-baryon systems, which depend on two unknown coupling constants $C_{a}$ and $C_{b}$.

\begin{tabular}{lccccc}
\hline \hline State & $J^{P}$ & $V$ & State & $J^{P}$ & $V$ \\
\hline $\bar{D} \Sigma_{c}$ & $1 / 2^{-}$ & $C_{a}$ & $\Xi_{c c} \Sigma_{c}$ & $0^{+}$ & $C_{a}+\frac{2}{3} C_{b}$ \\
& & & & $1^{+}$ & $C_{a}-\frac{2}{9} C_{b}$ \\
$\bar{D} \Sigma_{c}^{*}$ & $3 / 2^{-}$ & $C_{a}$ & $\Xi_{c c} \Sigma_{c}^{*}$ & $1^{+}$ & $C_{a}+\frac{5}{9} C_{b}$ \\
& & & & $2^{+}$ & $C_{a}-\frac{1}{3} C_{b}$ \\
$\bar{D}^{*} \Sigma_{c}$ & $1 / 2^{-}$ & $C_{a}-\frac{4}{3} C_{b}$ & $\Xi_{c c}^{*} \Sigma_{c}$ & $1^{+}$ & $C_{a}-\frac{10}{9} C_{b}$ \\
& $3 / 2^{-}$ & $C_{a}+\frac{2}{3} C_{b}$ & & $2^{+}$ & $C_{a}+\frac{2}{3} C_{b}$ \\
$\bar{D}^{*} \Sigma_{c}^{*}$ & $1 / 2^{-}$ & $C_{a}-\frac{5}{3} C_{b}$ & $\Xi_{c c}^{*} \Sigma_{c}^{*}$ & $0^{+}$ & $C_{a}-\frac{5}{3} C_{b}$ \\
& $3 / 2^{-}$ & $C_{a}-\frac{2}{3} C_{b}$ & & $1^{+}$ & $C_{a}-\frac{11}{9} C_{b}$ \\
& & & & $2^{+}$ & $C_{a}-\frac{1}{3} C_{b}$ \\
& $5 / 2^{-}$ & $C_{a}+C_{b}$ & & $3^{+}$ & $C_{a}+C_{b}$ \\
\hline \hline
\end{tabular}




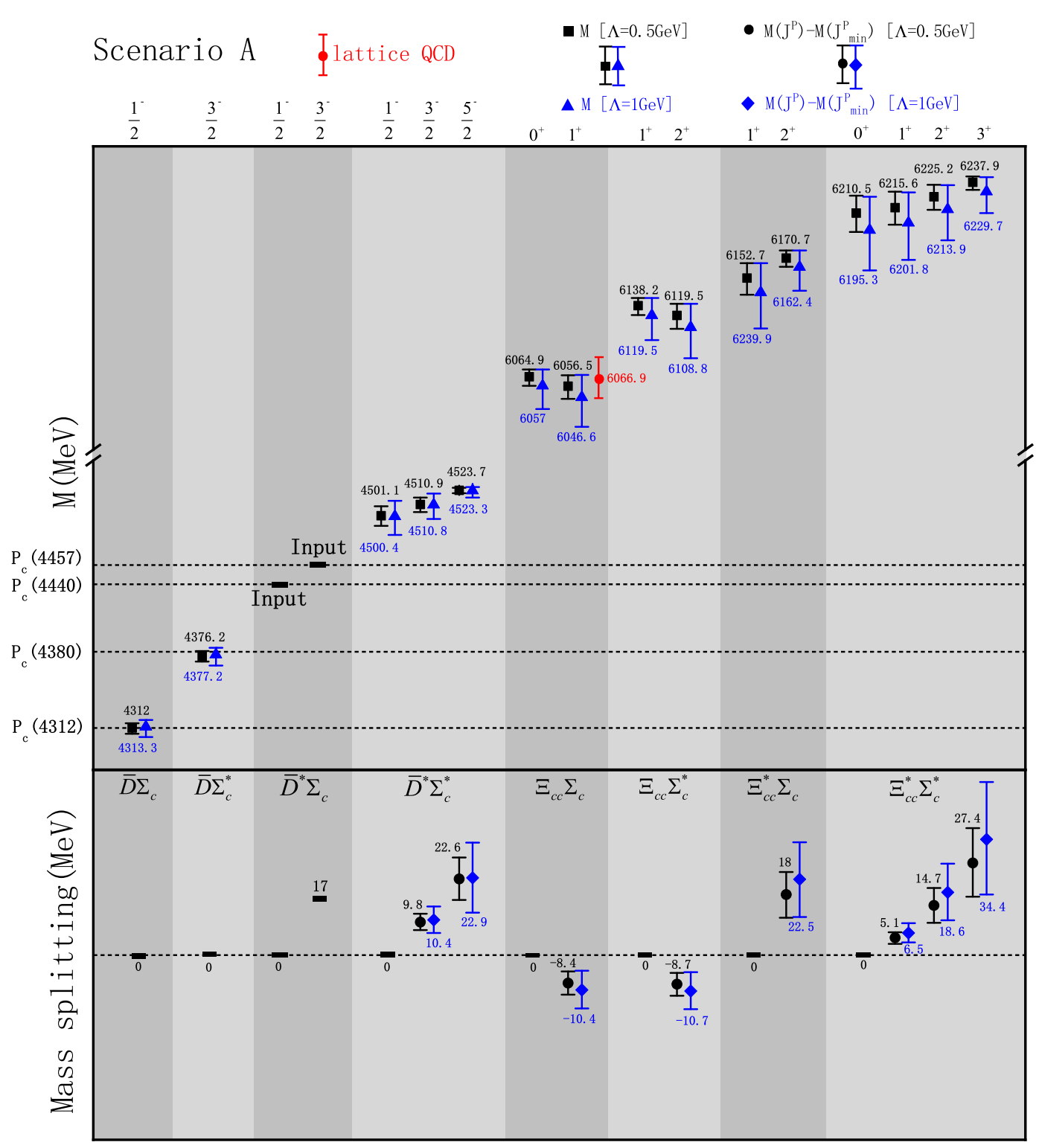

FIG. 1. Predicted masses (upper panel) and mass splittings between members of the multiplets (lower panel) of the pentaquark states and hexaquark (dibaryon) states in scenario A, in which the $P_{c}(4440) / P_{c}(4457)$ is identified with the $J=\frac{1}{2} / \frac{3}{2} \bar{D}^{*} \Sigma_{c}$ molecule. The dotted horizontal lines indicate the central experimental masses of the $P_{c}(4312), P_{c}(4380), P_{c}(4440)$, and $P_{c}(4457)$. The black squares and solid dots denote the results obtained with $\Lambda=0.5 \mathrm{GeV}$, while the blue triangles and diamonds denote the results obtained with $\Lambda=1 \mathrm{GeV}$. The numbers given in the figure are the central values. The uncertainties are obtained by assuming that HQS and HAD breakings are of the order of $15 \%$ and $25 \%$, respectively. The lattice QCD result for the $1^{+} \Xi_{c c} \Sigma_{c}$ state is calculated using the binding energy of Ref. [38] and the experimental masses for $\Xi_{c c}$ and $\Sigma_{c}$.

$J=1 / 2$ and $J=3 / 2$ doubly charmed baryons. ${ }^{3}$ For the present work, we will settle on a $25 \%$ error for the dibaryon potentials, yet we notice that for errors below $40 \%$ our predictions will remain qualitatively the same.

To obtain concrete predictions we have to solve a nonrelativistic bound-state equation with the contact-range

\footnotetext{
${ }^{3}$ For the pentaquark-dibaryon relation, a HADS violation of (25-30)\% suffices to postdict the $J^{P}=1^{+}$dibaryon at the location given by the lattice-QCD result [38]. This is compatible with the violations deduced from the mass splittings.
}

potentials of Table I. If we work in momentum space, we simply solve the Lippmann-Schwinger equation for the bound-state pole:

$$
\phi(k)+\int \frac{d^{3} p}{(2 \pi)^{3}}\langle k|V| p\rangle \frac{\phi(p)}{B+\frac{p^{2}}{2 \mu}}=0,
$$

where $\phi(k)$ is the vertex function, $B$ the binding energy, and $\mu$ the reduced mass. To solve this equation, we first regularize the potential 


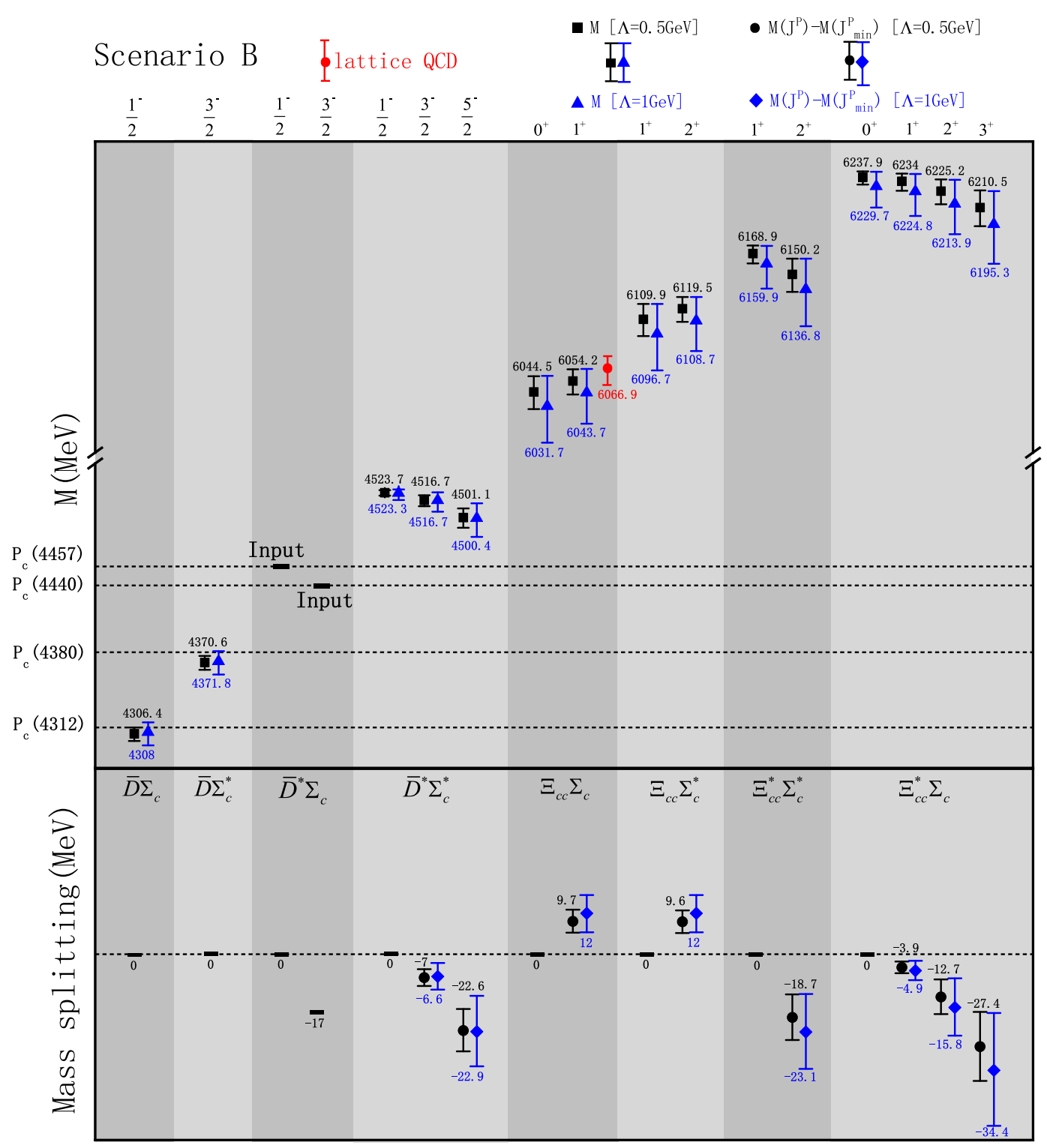

FIG. 2. The same as Fig. 1, but for scenario B, in which the $P_{c}(4440) / P_{c}(4457)$ is identified with the $J=\frac{3}{2} / \frac{1}{2} \bar{D}^{*} \Sigma_{c}$ molecule.

$$
\left\langle p\left|V_{\Lambda}\right| p^{\prime}\right\rangle=C(\Lambda) f\left(\frac{p}{\Lambda}\right) f\left(\frac{p^{\prime}}{\Lambda}\right)
$$

with $\Lambda$ the cutoff, $f(x)$ a regulator, and $C$ the linear combination of the $C_{a}$ and $C_{b}$ couplings corresponding to the molecular state we are interested in. Notice that this coupling depends on the cutoff. This is necessary if we want to properly renormalize the calculations; i.e., we want the predictions to depend on the cutoff only moderately. Cutoff variations represent the uncertainty coming from subleading terms that we have not explicitly taken into account, e.g., pion exchanges. This uncertainty refers to the physics of the light-quark degrees of freedom and is independent from the HQSS and HADS uncertainties. We will choose a Gaussian regulator $f(x)=e^{-x^{2}}$, and for the cutoff we will use the range $\Lambda=(0.5-1) \mathrm{GeV}$. The heavy-hadron masses are taken from the PDG [50] with the exception of the $\Xi_{c c}^{*}$, which is deduced from the HADS relation $m_{\Xi_{c c}^{*}}-m_{\Xi_{c c}}=3\left(m_{D^{*}}-m_{D}\right) / 4[45]$.

We still have to determine the couplings $C_{a}$ and $C_{b}$. For this, we notice that if the $P_{c}(4440)$ and $P_{c}(4457)$ are indeed $\bar{D}^{*} \Sigma_{c}$ bound states, their contact-range potentials read

$$
\begin{aligned}
& V\left(\bar{D}^{*} \Sigma_{c}, J=\frac{1}{2}\right)=C_{a}-\frac{4}{3} C_{b}, \\
& V\left(\bar{D}^{*} \Sigma_{c}, J=\frac{3}{2}\right)=C_{a}+\frac{2}{3} C_{b} .
\end{aligned}
$$

Since the spins of the hidden-charm pentaquarks are not experimentally known yet, there are two possible 
identifications: The $P_{c}(4440)$ is the spin- $1 / 2$ state and the $P_{c}(4457)$ the spin-3/2 one (scenario A), or vice versa (scenario B) [4]. In both scenarios, we arrive at the conclusion that the ten possible triply charmed dibaryons bind, with binding energies of (10-50) MeV; see Figs. 1 and 2 . The uncertainties are generated by randomly varying $C_{a}$ and $C_{b}$ around their central values within the range of the expected breakings of HQSS (15\%) and HADS (25\%).

One immediately notices that there is a strong correlation between the ordering of the triply charmed dibaryons and that of the pentaquark states, particularly that of the $P_{c}(4440)$ and $P_{c}(4457)$, as shown in the lower panels in Figs. 1 and 2. For instance, if the $0^{+} \Sigma_{c} \Xi_{c c}$ state had a larger mass than its $1^{+}$counterpart, then scenario A would be preferred. The difference in their binding energies is about $10 \mathrm{MeV}$, which might be achievable in the lattice. In addition, one can study (one of) the three extra multiplets to confirm the conclusion. For instance, a decrease of the mass as a function of the spin in the $\Xi_{c c} \Sigma_{c}\left(0^{+}, 1^{+}\right)$multiplet and an increase in the $\Xi_{c c}^{*} \Sigma_{c}\left(1^{+}, 2^{+}\right)$multiplet will both be unambiguous signals that scenario $\mathrm{A}$ is preferred.

More concretely, from Table I and assuming that the $C_{b}$ coupling is perturbative, we arrive at the relation

$$
\begin{aligned}
M\left(1^{+}\right)-M\left(0^{+}\right) & =-\frac{4}{9}\left(M\left(\frac{3^{-}}{2}\right)-M\left(\frac{1}{2}^{-}\right)\right) \\
& \approx \mp(7.6 \pm 1.9) \mathrm{MeV},
\end{aligned}
$$

with the $\mp$ sign, respectively, for scenarios A and B. We have used the experimental pentaquark masses to obtain the number on the second line (with the error corresponding to the HADS uncertainty). It is worth noticing that, in our numerical study with $\Lambda=0.5 \mathrm{GeV}$, we obtain $M\left(1^{+}\right)$$M\left(0^{+}\right)=-8.4_{-3.5}^{+3.4}$ and $+9.7_{-3.4}^{+3.4} \mathrm{MeV}$ for scenarios $\mathrm{A}$ and $\mathrm{B}$, respectively. Though the exact splitting may vary, the spectral ordering of Eq. (9) is found to survive even for larger breakdowns of HADS, specifically up to $39 \%$ after which a few dibaryon states unbind. If this unlikely possibility were to be realized, the symmetry relations between pentaquarks and dibaryons can still be formulated in terms of the scattering lengths of these systems.

In summary, we have predicted the full spectrum of S-wave triply-charmed dibaryons $\left(\Xi_{c c}^{(*)} \Sigma_{c}^{(*)}\right.$ molecules) within the framework of a contact-range EFT utilizing HQSS and HADS, assuming that the $P_{c}(4312), P_{c}(4440)$, and $P_{c}(4457)$ are hadronic molecules. More importantly, we pointed out a model-independent way to determine the spins of the pentaquarks from lattice QCD simulations of the dibaryons. The binding energies of the triply charmed dibaryons are predicted to lie in the (10-50) MeV range, where the details depend on the cutoff and the spins of the pentaquarks. Among them, we predict a $1^{+} \Xi_{c c} \Sigma_{c}$ dibaryon with a binding energy of (15-30) $\mathrm{MeV}$, to be compared with $(8 \pm 17) \mathrm{MeV}$ in the lattice [38]. The spin splitting of the dibaryon masses together with HADS, i.e., Eq. (9), indicates that a lattice-QCD calculation of the dibaryon spectrum will provide a model-independent determination of the quantum numbers of the LHCb pentaquarks. The recent calculation of Ref. [38] implies that such studies are within reach of state-of-the-art lattice-QCD simulations. Of course, the previous conclusions lean on the assumption that the pentaquarks are molecular: If they were largely nonmolecular, the relations derived here would not necessarily hold, thus providing a way to falsify the molecular hypothesis. In this sense, future lattice-QCD simulations of the dibaryons will provide a test of not only their symmetry relation with the pentaquarks, but also of the molecular nature of the latter.

\section{ACKNOWLEDGMENTS}

This work is partly supported by the National Natural Science Foundation of China under Grants No. 11735003, No. 11975041, and No. 11961141004, the fundamental Research Funds for the Central Universities, and the Thousand Talents Plan for Young Professionals.
[1] R. Aaij et al. (LHCb Collaboration), Phys. Rev. Lett. 122, 222001 (2019).

[2] H.-X. Chen, W. Chen, and S.-L. Zhu, Phys. Rev. D 100, 051501 (2019).

[3] R. Chen, Z.-F. Sun, X. Liu, and S.-L. Zhu, Phys. Rev. D 100, 011502 (2019).

[4] M.-Z. Liu, Y.-W. Pan, F.-Z. Peng, M. S. Sánchez, L.-S. Geng, A. Hosaka, and M. P. Valderrama, Phys. Rev. Lett. 122, 242001 (2019).

[5] F.-K. Guo, H.-J. Jing, U.-G. Meißner, and S. Sakai, Phys. Rev. D 99, 091501 (2019).
[6] C. W. Xiao, J. Nieves, and E. Oset, Phys. Rev. D 100, 014021 (2019).

[7] Y. Shimizu, Y. Yamaguchi, and M. Harada, arXiv:1904 00587.

[8] Z.-H. Guo and J. A. Oller, Phys. Lett. B 793, 144 (2019).

[9] J.-J. Wu, R. Molina, E. Oset, and B. S. Zou, Phys. Rev. Lett. 105, 232001 (2010).

[10] J.-J. Wu, R. Molina, E. Oset, and B. S. Zou, Phys. Rev. C 84, 015202 (2011).

[11] C. W. Xiao, J. Nieves, and E. Oset, Phys. Rev. D 88, 056012 (2013). 
[12] M. Karliner and J. L. Rosner, Phys. Rev. Lett. 115, 122001 (2015).

[13] W. L. Wang, F. Huang, Z. Y. Zhang, and B. S. Zou, Phys. Rev. C 84, 015203 (2011).

[14] Z.-C. Yang, Z.-F. Sun, J. He, X. Liu, and S.-L. Zhu, Chin. Phys. C 36, 6 (2012).

[15] M. I. Eides, V. Y. Petrov, and M. V. Polyakov, Mod. Phys. Lett. A 35, 2050151 (2020).

[16] A. Ali and A. Ya. Parkhomenko, Phys. Lett. B 793, 365 (2019).

[17] Z.-G. Wang, Int. J. Mod. Phys. A 35, 2050003 (2020).

[18] J.-B. Cheng and Y.-R. Liu, Phys. Rev. D 100, 054002 (2019).

[19] Y. Yamaguchi, H. Garcia-Tecocoatzi, A. Giachino, A. Hosaka, E. Santopinto, S. Takeuchi, and M. Takizawa, Phys. Rev. D 101, 091502 (2020).

[20] M. P. Valderrama, Phys. Rev. D 100, 094028 (2019).

[21] M.-Z. Liu, T.-W. Wu, M. S. Sánchez, M. P. Valderrama, L.-S. Geng, and J.-J. Xie, arXiv:1907.06093.

[22] R. Aaij et al. (LHCb Collaboration), Phys. Rev. Lett. 115, 072001 (2015).

[23] T. Sugiura, Y. Ikeda, and N. Ishii, Proc. Sci., LATTICE2018 (2019) 093 [arXiv:1905.02336].

[24] U. Skerbis and S. Prelovsek, Phys. Rev. D 99, 094505 (2019).

[25] N. Isgur and M. B. Wise, Phys. Lett. B 232, 113 (1989).

[26] N. Isgur and M. B. Wise, Phys. Lett. B 237, 527 (1990).

[27] J. Nieves and M. P. Valderrama, Phys. Rev. D 86, 056004 (2012).

[28] F.-K. Guo, C. Hidalgo-Duque, J. Nieves, and M. P. Valderrama, Phys. Rev. D 88, 054014 (2013).

[29] C. W. Xiao, J. Nieves, and E. Oset, Phys. Lett. B 799, 135051 (2019).

[30] F.-Z. Peng, M.-Z. Liu, Y.-W. Pan, M. S. Sánchez, and M. P. Valderrama, arXiv:1907.05322.
[31] A. E. Bondar, A. Garmash, A. I. Milstein, R. Mizuk, and M. B. Voloshin, Phys. Rev. D 84, 054010 (2011).

[32] T. Mehen and J. W. Powell, Phys. Rev. D 84, 114013 (2011).

[33] F.-K. Guo, C. Hidalgo-Duque, J. Nieves, and M. P. Valderrama, Phys. Rev. D 88, 054007 (2013).

[34] M. J. Savage and M. B. Wise, Phys. Lett. B 248, 177 (1990).

[35] S. Sakai, H.-J. Jing, and F.-K. Guo, Phys. Rev. D 100, 074007 (2019).

[36] M.-L. Du, V. Baru, F.-K. Guo, C. Hanhart, U.-G. Meißner, J. A. Oller, and Q. Wang, Phys. Rev. Lett. 124, 072001 (2020).

[37] M.-Z. Liu, F.-Z. Peng, M. S. Sánchez, and M. P. Valderrama, Phys. Rev. D 98, 114030 (2018).

[38] P. Junnarkar and N. Mathur, Phys. Rev. Lett. 123, 162003 (2019).

[39] M.-Z. Liu, T.-W. Wu, J.-J. Xie, M. P. Valderrama, and L.-S. Geng, Phys. Rev. D 98, 014014 (2018).

[40] A. F. Falk and M. E. Luke, Phys. Lett. B 292, 119 (1992).

[41] P. L. Cho, Nucl. Phys. B396, 183 (1993); B421, 683(E) (1994).

[42] M. P. Valderrama, Eur. Phys. J. A 56, 109 (2020).

[43] M. P. Valderrama, Phys. Rev. D 85, 114037 (2012).

[44] J.-X. Lu, L.-S. Geng, and M. P. Valderrama, Phys. Rev. D 99, 074026 (2019).

[45] J. Hu and T. Mehen, Phys. Rev. D 73, 054003 (2006).

[46] M. Padmanath, R. G. Edwards, N. Mathur, and M. Peardon, Phys. Rev. D 91, 094502 (2015).

[47] Y.-C. Chen and T.-W. Chiu (TWQCD Collaboration), Phys. Lett. B 767, 193 (2017).

[48] C. Alexandrou and C. Kallidonis, Phys. Rev. D 96, 034511 (2017).

[49] N. Mathur and M. Padmanath, Phys. Rev. D 99, 031501 (2019).

[50] M. Tanabashi et al. (Particle Data Group), Phys. Rev. D 98, 030001 (2018). 